Original Research Paper

\title{
About Nano Fusion and Dynamic Fusion
}

\author{
${ }^{1}$ Florian Ion T. Petrescu and ${ }^{2}$ John Kaiser Calautit \\ ${ }^{I}$ Department of Theory of Mechanisms and Robots, Bucharest Polytechnic University, Bucharest, Romania \\ ${ }^{2}$ Department of Mechanical Engineering, University of Sheffield, Sheffield, United Kingdom
}

Article history

Received: 02-26-2016

Revised: 02-27-2016

Accepted: 02-29-2016

Corresponding author:

Florian Ion T. Petrescu

Department of Theory of

Mechanisms and Robots,

Bucharest Polytechnic

University, Bucharest, Romania

Email: petrescuflorian@yahoo.com

\begin{abstract}
Hot fusion is currently a difficult goal to accomplish due to the high temperatures required, which are difficult to achieve and also to be maintained. For these reason, it is much easier to try to achieve cold fusion, or a combined method. In this paper, the author will briefly present some original relationships for setting up a theoretical model for cold fusion. It will be determined the radius of a moving elementary particle and will be calculated the potential energy of the two adjacent particles. In addition, the necessary speed of the accelerated particles when they will collide to start cold fusion will be determined. The radius of an electron or a nucleus at rest is close to nano sizes. Because of this, static, the fusion working with nanoparticles. It was evaluated that dynamic nanoparticles dimensions are much smaller than when they are at rest.
\end{abstract}

Keywords: Nanoparticles, Fusion, Cold Fusion, Dynamic Cold Fusion, Moving Particle, Adjacent Particles, Moving Particle Radius, Potential Energy, Accelerated Particles Speed

\section{Introduction}

In general, for determining the size of atomic and subatomic particles their static diameters (ie when the particle is at rest) are used, which is calculated by various approximated methods (Halliday and Robert, 1966). These dimensions are of the order of nano, pico or slightly lower size. The real phenomena occur when these particles are in dynamically movement and it is therefore necessary to know the real dimensions of the particles in movement. This paper aims to accomplish this. The parameters required for the fusion of two Deuterium particles will be calculated. The known nano fusion parameters will be replaced with dynamic fusion parameters. The first one will determine the necessary speed of the accelerated particles needed to start cold fusion when they collide. The second one will determine the radius of a moving Deuterium particle. The third one will calculate the potential energy of the two adjacent Deuterium particles on fusion. This is the kinetic energy that must reach a Deuterium particle accelerated to produce fission by collision.

\section{Materials and Methods}

Any elementary moving particle possesses the kinetic energy given by the relationship 1 (composed by two components: the kinetic energy of motion translational and rotational kinetic energy of motion):

$$
E_{c}=\frac{1}{2} m \cdot v^{2}+\frac{1}{2} J \cdot \omega^{2}
$$

The mass of particle is determined with the Lorentz relationship 2 (Lorentz):

$$
m=\frac{m_{0} \cdot c}{\sqrt{c^{2}-v^{2}}}
$$

Mechanical moment of inertia (mass) of particle (around its axis of rotation) is determined by the relationship 3:

$$
J=\frac{2}{5} m \cdot R^{2}
$$

This is the mass moment of inertia of a sphere (see the Fig. 1).

Using the forms 2 and 3 the relationship 1 takes the forms 4:

$$
\left\{\begin{array}{l}
E_{c}=\frac{1}{2} m \cdot v^{2}+\frac{1}{2} \cdot \frac{2}{5} \cdot m \cdot R^{2} \cdot \omega^{2} \\
E_{c}=\frac{1}{2} \frac{m_{0} \cdot c \cdot v^{2}}{\sqrt{c^{2}-v^{2}}}+\frac{1}{2} \cdot \frac{2}{5} \cdot \frac{m_{0} \cdot c \cdot R^{2}}{\sqrt{c^{2}-v^{2}}} \cdot \omega^{2}
\end{array}\right.
$$




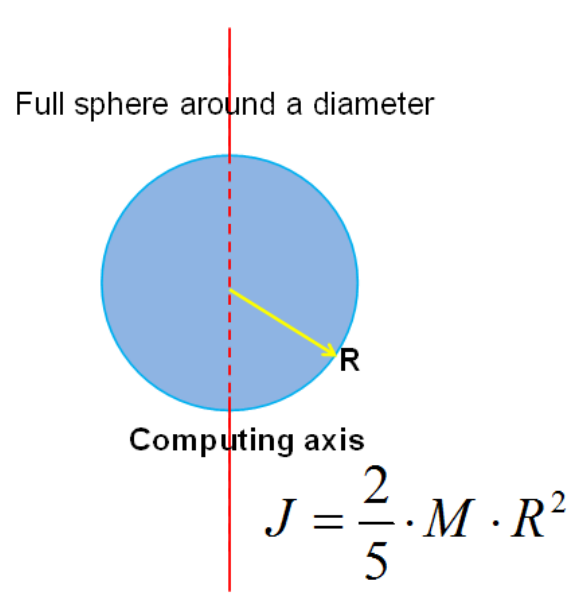

Fig. 1. Mass moment of inertia to a full sphere, determined around a diameter

Pulse of the particle is written using the relation 5:

$$
p=m \cdot v=\frac{m_{0} \cdot c \cdot v}{\sqrt{c^{2}-v^{2}}}
$$

The wavelength associated with the particle can be determined with the relationship 6 (according to Louis de Broglie the pulse is conserved):

$\lambda=\frac{h}{p}=\frac{h \cdot \sqrt{c^{2}-v^{2}}}{m_{0} \cdot c \cdot v}$

Wave frequency associated with the particle is determining by relationship 7 :

$$
\gamma=\frac{c}{\lambda}=\frac{c \cdot m_{0} \cdot c \cdot v}{h \cdot \sqrt{c^{2}-v^{2}}}=\frac{m_{0} \cdot c^{2} \cdot v}{h \cdot \sqrt{c^{2}-v^{2}}}
$$

The angular velocity of the particle and its square can be calculated with the relationships 8 :

$$
\left\{\begin{array}{l}
\omega=2 \pi \gamma=\frac{2 \pi \cdot m_{0} \cdot c^{2} \cdot v}{h \cdot \sqrt{c^{2}-v^{2}}} \\
\omega^{2}=\frac{4 \pi^{2} \cdot m_{0}^{2} \cdot c^{4} \cdot v^{2}}{h^{2} \cdot\left(c^{2}-v^{2}\right)}
\end{array}\right.
$$

Using 8 the relationship 4 takes the forms 9:

$$
\left\{\begin{array}{l}
E_{c}=\frac{1}{2} \frac{m_{0} \cdot c \cdot v^{2}}{\sqrt{c^{2}-v^{2}}}+ \\
+\frac{1}{2} \cdot \frac{2}{5} \cdot \frac{m_{0} \cdot c \cdot R^{2}}{\sqrt{c^{2}-v^{2}}} \cdot \frac{4 \pi^{2} \cdot m_{0}^{2} \cdot c^{4} \cdot v^{2}}{h^{2} \cdot\left(c^{2}-v^{2}\right)} \\
E_{c}=\frac{1}{2} \frac{m_{0} \cdot c \cdot v^{2}}{\sqrt{c^{2}-v^{2}}} \cdot\left[1+\frac{8}{5} R^{2} \cdot \pi^{2} \frac{m_{0}^{2} \cdot c^{4}}{h^{2} \cdot\left(c^{2}-v^{2}\right)}\right]
\end{array}\right.
$$

The kinetic energy of the moving particle can be determined and by the relationship 10 . From the total energy of the particle in movement, subtract the total energy of the particle at rest (potential energy):

$$
\begin{aligned}
& E_{c}=E-E_{0}=m \cdot c^{2}-m_{0} \cdot c^{2}= \\
& =m_{0} \cdot c^{2} \cdot \frac{c-\sqrt{c^{2}-v^{2}}}{\sqrt{c^{2}-v^{2}}}
\end{aligned}
$$

Identifying the relationships 9 and 10 are obtained the expressions 11 which can determine the radius of an elementary moving particle:

$$
\left\{\begin{array}{l}
\frac{1}{2} \frac{m_{0} \cdot c \cdot v^{2}}{\sqrt{c^{2}-v^{2}}} \cdot\left[1+\frac{8}{5} R^{2} \cdot \pi^{2} \frac{m_{0}^{2} \cdot c^{4}}{h^{2} \cdot\left(c^{2}-v^{2}\right)}\right]= \\
=m_{0} \cdot c^{2} \cdot \frac{c-\sqrt{c^{2}-v^{2}}}{\sqrt{c^{2}-v^{2}}} \\
R=\sqrt{\frac{10}{8}} \cdot \frac{h}{\pi \cdot m_{0} \cdot c^{2}} \cdot \\
\frac{\sqrt{c^{2}-v^{2}} \cdot \sqrt{c^{2}-\frac{v^{2}}{2}-c \cdot \sqrt{c^{2}-v^{2}}}}{v} \\
R=\sqrt{\frac{10}{8}} \cdot \frac{h \cdot \sqrt{c^{2}-v^{2}} \cdot \sqrt{c^{2}-\frac{v^{2}}{2}-c \cdot \sqrt{c^{2}-v^{2}}}}{\pi \cdot m_{0} \cdot c^{2} \cdot v}
\end{array}\right.
$$

It is known and the expression of potential energy between two adjacent particles (electrostatic potential energy), which should be the energy with that a particle needs to be accelerated before to collide (relationships 12, Fig. 2), (Petrescu, 2012). This electrostatic potential energy must to be the same with the (final) kinetic energy of motion translational of accelerated particle $E_{p}=1 / 2 \mathrm{mv}^{2}$ :

$$
\left\{\begin{array}{l}
E_{p}=\frac{1}{4 \pi \cdot \varepsilon_{0}} \cdot \frac{q_{1} \cdot q_{2}}{d_{12}}=\frac{q_{1} \cdot q_{2}}{8 \pi \cdot \varepsilon_{0} \cdot R} \\
E_{p}=\frac{1}{2} m \cdot v^{2}
\end{array}\right.
$$

The radius of Deuterium at rest (without motion), was determined in Fig. 2 according to the following relationship 13:

$$
\left\{\begin{array}{l}
R_{D}=r_{0} \cdot A^{1 / 3} \\
r_{0}=1,45 E-15[\mathrm{~m}] \text { the average radius } \\
\text { of a nucleon fixed } \\
\mathrm{A}=\text { the atomic mass }
\end{array}\right.
$$




$$
\begin{aligned}
& d_{12 D}=2 \cdot R_{D}=2 \cdot 1.8268855223476 \cdot 10^{-15}[\mathrm{~m}]= \\
& =3.6537710446952 \cdot 10^{-15}[\mathrm{~m}]= \\
& \approx 3.653771 \cdot 10^{-15}[\mathrm{~m}]
\end{aligned}
$$

Fig. 2. Two adjacent particles of deuterium

With relationship 11 we can determine with very high accuracy the radius of a Deuteron or any other elementary moving particle, as a function of its velocity, v. The kinetic energy produced by the rotation of the particle is expressed by the relationship 14:

$$
\left\{\begin{array}{l}
E_{c \omega}=\frac{1}{2} \cdot \frac{2}{5} \cdot m \cdot R^{2} \cdot \omega^{2}= \\
=\frac{2}{10} \cdot m \cdot R^{2} \cdot \frac{4 \pi^{2} \cdot m^{2} \cdot c^{2} \cdot v^{2}}{h^{2}}= \\
=\frac{8}{10} \frac{\pi^{2} \cdot m^{3} \cdot c^{2} \cdot v^{2} \cdot R^{2}}{h^{2}}
\end{array}\right.
$$

In order to produce the fusion of two particles they must be brought close together with potential energy expressed by the relationship 12, where the electrostatic potential energy of particle was replaced by $E_{p}=1 / 2 m v^{2}$. For this reason the length of a particle radius (in movement) may be determined from relationship 12, taking the expression 15 :

$$
R=\frac{q_{1} \cdot q_{2}}{4 \pi \cdot \varepsilon_{0} \cdot m \cdot v^{2}}
$$

Using the relationship 15, expression 14 takes the form 16:

$$
E_{c \omega}=\frac{\left(q_{1} \cdot q_{2}\right)^{2} \cdot c^{2} \cdot m}{20 h^{2} \cdot \varepsilon_{0}^{2} \cdot v^{2}}
$$

The kinetic energy produced by the rotation of the particle (expressed by the relationship 16) may be written and by expression 17. From the total energy of the moving particle, subtract the kinetic energy produced by the translational motion of the particle and the total energy of the particle at rest (potential energy):

$$
E_{c \omega}=m \cdot c^{2}-\frac{1}{2} m \cdot v^{2}-m_{0} \cdot c^{2}
$$

Equaling expressions 16 and 17 obtains the equation 18 , in $\mathrm{v}^{2}$ : $m \cdot c^{2}-\frac{1}{2} m \cdot v^{2}-m_{0} \cdot c^{2}=\frac{\left(q_{1} \cdot q_{2}\right)^{2} \cdot c^{2} \cdot m}{20 h^{2} \cdot \varepsilon_{0}^{2} \cdot v^{2}}$

The equation 18 takes the forms 19-26:

$m c^{2}-m_{0} c^{2}=\frac{\left(q_{1} q_{2}\right)^{2} c^{2} m+10 m v^{4} h^{2} \varepsilon_{0}^{2}}{20 h^{2} \cdot \varepsilon_{0}^{2} \cdot v^{2}}$

$\frac{m_{0} c^{2}\left(c-\sqrt{c^{2}-v^{2}}\right)}{\sqrt{c^{2}-v^{2}}}=\frac{c m_{0}\left[\left(q_{1} q_{2}\right)^{2} c^{2}+10 h^{2} \varepsilon_{0}^{2} v^{4}\right]}{20 h^{2} \cdot \varepsilon_{0}^{2} \cdot v^{2} \sqrt{c^{2}-v^{2}}}$

$20 h^{2} \varepsilon_{0}^{2} v^{2}\left(c^{2}-c \sqrt{c^{2}-v^{2}}\right)=\left(q_{1} q_{2}\right)^{2} c^{2}+10 h^{2} \varepsilon_{0}^{2} v^{4}$

$\left\{\begin{array}{l}20 h^{2} \varepsilon_{0}^{2} v^{2} c^{2}-20 h^{2} \varepsilon_{0}^{2} v^{2} c \sqrt{c^{2}-v^{2}}= \\ =\left(q_{1} q_{2}\right)^{2} c^{2}+10 h^{2} \varepsilon_{0}^{2} v^{4}\end{array}\right.$

$\left\{\begin{array}{l}20 h^{2} \varepsilon_{0}^{2} v^{2} c^{2}-\left(q_{1} q_{2}\right)^{2} c^{2}-10 h^{2} \varepsilon_{0}^{2} v^{4}= \\ =20 h^{2} \varepsilon_{0}^{2} v^{2} c \sqrt{c^{2}-v^{2}}\end{array}\right.$

$\left\{\left(20 h^{2} \varepsilon_{0}^{2} v^{2} c^{2}-\left(q_{1} q_{2}\right)^{2} c^{2}-10 h^{2} \varepsilon_{0}^{2} v^{4}\right)^{2}=\right.$

$\left\{=400 h^{4} \varepsilon_{0}^{4} v^{4} c^{2}\left(c^{2}-v^{2}\right)\right.$

$\left\{\begin{array}{l}100 h^{4} \varepsilon_{0}^{4} x^{4}+200 h^{4} \varepsilon_{0}^{4} c^{2} x^{3}+ \\ +20 h^{2} \varepsilon_{0}^{2} c^{2}\left(q_{1} q_{2}\right)^{2} x^{2}- \\ -40 h^{2} \varepsilon_{0}^{2} c^{4}\left(q_{1} q_{2}\right)^{2} x+\left(q_{1} q_{2}\right)^{4} c^{4}=0 \\ \text { with } x=v^{2}\end{array}\right.$

$\left\{\begin{array}{l}x^{4}+a x^{3}+b x^{2}-c x+d=0 \\ a=2 c^{2} \\ b=\frac{c^{2}\left(q_{1} q_{2}\right)^{2}}{5 h^{2} \varepsilon_{0}^{2}} \\ c=\frac{2 c^{4}\left(q_{1} q_{2}\right)^{2}}{5 h^{2} \varepsilon_{0}^{2}} \\ d=\frac{c^{4}\left(q_{1} q_{2}\right)^{4}}{100 h^{4} \varepsilon_{0}^{4}}\end{array}\right.$

Solving the equation 26 in $\mathrm{v}^{2}$ one obtains the value $\mathrm{v}$ for velocity of the accelerated particle required for fusion (expression 27):

$\left\{\begin{array}{l}v^{2}=4.784 \mathrm{E}+11\left[\mathrm{~m}^{2} / \mathrm{s}^{2}\right] \\ v=691664.8602[\mathrm{~m} / \mathrm{s}] \\ \beta=\mathrm{v} / \mathrm{c}=0.002307088\end{array}\right.$ 


\section{Results and Discussion}

First one should determine the necessary speed of the accelerated particles needed to start cold fusion when they will collide. It may use the Equation 26.

This speed has the value: $\mathrm{v}=691664.8602[\mathrm{~m} / \mathrm{s}]$

Second one could determine the radius of a moving deuterium particle using the relationship 28 (extract from 11):

$R=\sqrt{\frac{10}{8}} \cdot \frac{h \cdot \sqrt{c^{2}-v^{2}} \cdot \sqrt{c^{2}-\frac{v^{2}}{2}-c \cdot \sqrt{c^{2}-v^{2}}}}{\pi \cdot m_{0} \cdot c^{2} \cdot v}$

Third one may calculate the potential energy of the two adjacent Deuterium particles on fusion. This is the minimum translational kinetic energy that must reach a Deuterium particle accelerated to produce fission by collision (using the form 29 of relationship 12):

$$
E_{p}=\frac{q_{1} \cdot q_{2}}{8 \pi \cdot \varepsilon_{0} \cdot R}
$$

With $\mathrm{m}_{0}$ deuteron $=3.34524 \mathrm{E}-27[\mathrm{~kg}]$

$\mathrm{v}=691664.8602[\mathrm{~m} / \mathrm{s}]$

The radius of Deuteron at this velocity (calculated with relationship 28) takes the below value:

$\mathrm{RD}=1.91788 \mathrm{E}-19[\mathrm{~m}]($ dynamic at $\mathrm{v}=0.002307088 \mathrm{c})$

Static RD $=1.827 \mathrm{E}-15[\mathrm{~m}]$.

Potential energy has the below value:

$\mathrm{U}=\mathrm{Ep}=6.01333 \mathrm{E}-10[\mathrm{~J}]=3753521838[\mathrm{eV}]=$

$3753521.838[\mathrm{KeV}]=3753.521838[\mathrm{MeV}]=$

$3.753521838[\mathrm{GeV}]$

We need to accelerate a Deuteron with minimum $3.76 \mathrm{GeV}$ to prepare it and then one collide these accelerated Deuterium ions to start the fusion process. Today, cold fusion requires a storage and maintenance stage of the merger process somewhat similar to hot fusion, while hot fusion can't be achieved only at high temperatures, but also other complementary measures. For this reason we must speak about a combined method for fusion (De Ninno et al., 2002). Anyway, I would not conclude that "cold fusion" it is "much easier than hot fusion".

Expression 28 has the advantage to give the dynamic radius of one particle at any velocity except the two limits when $\mathrm{v}=\mathrm{c}$ or $\mathrm{v}=0$.
If $\mathrm{v}=\mathrm{c}$ the particle become a photon and one can use other equations.

\section{Conclusion}

Dynamic, the matter focuses!

Hot fusion is currently a difficult goal to accomplish. Hot fusion temperature required is huge and difficult to achieve and much less to be supported and maintained. For these reasons it is much easier to try to achieve cold fusion, or a combined method. The presented paper briefly shows some original relationships which can help to set up a theoretical model for cold fusion (combined fusion). One should determine the necessary speed of the accelerated particles so that when they will collide to start cold fusion. Then, it will determine the radius of a moving particle and calculate the potential energy of the two adjacent particles. A nucleus at rest is close to nano size. Because of this (static) the fusion working with nanoparticles. But it was shown that dynamic particles dimensions are much smaller than when they are at rest. We may speak about a dynamic fusion (and not a nano one). Two new relationships 27, 28 were introduced.

\section{Assumptions Used}

- The assumption that frequency of the particle associate wave is the same with rotation frequency of the particle (the rotation of the particle produces the associate wave) was used

- The assumption that expression of potential energy between two adjacent particles (electrostatic potential energy), should be the same with minimum translational kinetic energy with that a particle needs to be accelerated before its collision

\section{Importance}

With the new expression (28) introduced one can determine the radius of any elementary particle in movement.

In the (below) Table 1 it can see the radius of an electron in movement (determined with expression 28) in function of $\beta$ (where $\beta$ is the ratio between $\mathrm{v}$ and $\mathrm{c}$; expression 30):

$$
\beta=\frac{v}{c}
$$

Dynamic, at low or high velocities the matter focuses.

One can determine the value of average radius of an electron 1.09756E-13 [m] and its maximum value $1.83152 \mathrm{E}-13$ [m] obtained for $\beta=0.8$. 
Table 1. The electron radius in function of $\beta$

\begin{tabular}{llll}
\hline$\beta$ & 0.000009 & 0.00002 & 0.0001 \\
\hline$R[\mathrm{~m}]$ & $4.93 \mathrm{E}-16$ & $4.07 \mathrm{E}-16$ & $8.15 \mathrm{E}-17$ \\
$\beta$ & 0.001 & 0.01 & 0.1 \\
$\mathrm{R}[\mathrm{m}]$ & $3.05 \mathrm{E}-16$ & $3.05 \mathrm{E}-15$ & $3.04 \mathrm{E}-14$ \\
$\beta$ & 0.2 & 0.3 & 0.4 \\
$\mathrm{R}[\mathrm{m}]$ & $6.04 \mathrm{E}-14$ & $8.94 \mathrm{E}-14$ & $1.16 \mathrm{E}-13$ \\
$\beta$ & 0.5 & 0.6 & 0.7 \\
$\mathrm{R}[\mathrm{m}]$ & $1.41 \mathrm{E}-13$ & $1.62 \mathrm{E}-13$ & $1.78 \mathrm{E}-13$ \\
$\beta$ & 0.8 & 0.9 & 0.99 \\
$\mathrm{R}[\mathrm{m}]$ & $1.83 \mathrm{E}-13$ & $1.66 \mathrm{E}-13$ & $7.47 \mathrm{E}-14$ \\
$\beta$ & 0.999 & 0.9999 & 0.99999 \\
$\mathrm{R}[\mathrm{m}]$ & $2.61 \mathrm{E}-14$ & $8.51 \mathrm{E}-15$ & $2.71 \mathrm{E}-15$ \\
$\beta$ & 0.999999 & 0.9999999 & 0.99999999 \\
$\mathrm{R}[\mathrm{m}]$ & $8.62 \mathrm{E}-16$ & $2.72 \mathrm{E}-16$ & $8.63 \mathrm{E}-17$ \\
\hline
\end{tabular}

Table 2. The proton radius in function of $\beta$

\begin{tabular}{llll}
\hline$\beta$ & 0.000009 & 0.00002 & 0.0001 \\
\hline $\mathrm{R}[\mathrm{m}]$ & $2.68 \mathrm{E}-19$ & $2.21 \mathrm{E}-19$ & $4.43 \mathrm{E}-20$ \\
$\beta$ & 0.001 & 0.01 & 0.1 \\
$\mathrm{R}[\mathrm{m}]$ & $1.66 \mathrm{E}-19$ & $1.66 \mathrm{E}-18$ & $1.65 \mathrm{E}-17$ \\
$\beta$ & 0.2 & 0.3 & 0.4 \\
$\mathrm{R}[\mathrm{m}]$ & $3.29 \mathrm{E}-17$ & $4.87 \mathrm{E}-17$ & $6.36 \mathrm{E}-17$ \\
$\beta$ & 0.5 & 0.6 & 0.7 \\
$\mathrm{R}[\mathrm{m}]$ & $7.71 \mathrm{E}-17$ & $8.86 \mathrm{E}-17$ & $9.69 \mathrm{E}-17$ \\
$\beta$ & 0.8 & 0.9 & 0.99 \\
$\mathrm{R}[\mathrm{m}]$ & $9.97 \mathrm{E}-17$ & $9.08 \mathrm{E}-17$ & $4.06 \mathrm{E}-17$ \\
$\beta$ & 0.999 & 0.9999 & 0.99999 \\
$\mathrm{R}[\mathrm{m}]$ & $1.42 \mathrm{E}-17$ & $4.63 \mathrm{E}-18$ & $1.48 \mathrm{E}-18$ \\
$\beta$ & 0.999999 & 0.9999999 & 0.99999999 \\
$\mathrm{R}[\mathrm{m}]$ & $4.69 \mathrm{E}-19$ & $1.48 \mathrm{E}-19$ & $4.70 \mathrm{E}-20$ \\
\hline
\end{tabular}

In the same mode one determines the radius of a proton in movement (Table 2).

One can determine the value of average radius of a proton (or neutron) $5.9779 \mathrm{E}-17[\mathrm{~m}]$ and its maximum value $9.97547 \mathrm{E}-17[\mathrm{~m}] \cong 1 \mathrm{E}-16[\mathrm{~m}]$ obtained for $\beta=0.8$.

\section{Observations (Very Important)}

Even the electron mass is smaller than the proton or neutron (nucleon) mass, the electron size is greater than the nucleon size.

When the number of nucleons increases (when the atomic mass $\mathrm{A}$, the mass of nucleus, increases) the nucleus size decreases. In a deuterium nucleus we have two nucleons (a proton and a neutron). These are the results of the new expression 28 indicating a matter compression inversely proportional to the mass (see the below values).

$\mathrm{m}_{0}$ electron $=9.11 \mathrm{E}-31[\mathrm{~kg}]=>\mathrm{Re}=1.09756 \mathrm{E}-13[\mathrm{~m}]$; $\mathrm{m}_{0}$ proton $=1.67262 \mathrm{E}-27[\mathrm{~kg}]=>\mathrm{Rp}=5.9779 \mathrm{E}-17[\mathrm{~m}] ;$ $\mathrm{m}_{0}$ deuteron $=3.3452 \mathrm{E}-27[\mathrm{~kg}]=>\mathrm{R}_{\mathrm{D}}=2.9889 \mathrm{E}-17[\mathrm{~m}]$.

For fusion, cold or hot, one must use positive ions of Deuterium called Deuterons, to have the possibility to accelerate them.

\section{Utilization and comments}

The average value of a Deuteron radius is 2.98895E-17 [m]. With this Deuteron radius value, using the expression (29) one may determine the translational kinetic energy with that needs to accelerate this particle before collide it:

$$
\begin{aligned}
& \mathrm{U}=\mathrm{Ep}=3.85849 \mathrm{E}-12[\mathrm{~J}]=24084723.86[\mathrm{eV}]= \\
& 24084.72386[\mathrm{keV}]=24.08472386[\mathrm{MeV}]
\end{aligned}
$$

In this way we can eliminate the second (dynamic) assumption.

Or $U=3.76[\mathrm{GeV}]$ with the New Dynamic Theory

Or $\mathrm{U}=24[\mathrm{MeV}]$ using an average value for the Deuteron radius.

However, the final word will be said only by more practical experiments. The authors think that real process is one dynamic and recommend a minimum $\mathrm{U}$ $=3.76 \mathrm{GeV}$ ).

\section{Acknowledgement}

This text was acknowledged and appreciated by Assoc. Pro. Taher M. Abu-Lebdeh, North Carolina A and $\mathrm{T}$ State Univesity, United States, Samuel P. Kozaitis, Professor and Department Head at Electrical and Computer Engineering, Florida Institute of Technology, United States, Mauro Pravettoni, PhD MEng MSc Researcher, CH-6814 Lamone, Switzerland and Prof. Antonio Apicella, Materials Engineer, full professor of Materials Science and Technology, Director of the International Master in "Design Driven Sustainable Innovation", Advanced Material Lab, Dept of Architecture and Industrial Design, Second University of Naples, Italy.

\section{Author's Contributions}

Florian Ion T. Petrescu: Established the new calculation relationships presented in the paper.

John Kaiser Calautit: Participated in all experiments, coordinated the data-analysis and contributed to the writing of the manuscript and English correction.

\section{Ethics}

This article is original and contains unpublished material. The corresponding author approved the manuscript and confirms that no ethical issues involved. 


\section{References}

De Ninno, A., A. Frattolillo, A. Rizzo and E. Del Giudice, 2002. Preparata, Experimental Evidence of 4 He Production in a cold fusion experiment. ENEA Technical Report, RT2002/41/FUS.

Halliday, D. and R. Robert, 1966. Physics, Part II. 1st Edn., John Wiley and Sons, Inc., New York.

Louis de Broglie, Wikipedia. https://en.wikipedia.org/wiki/Louis_de_Broglie

Lorentz transformation, - - Wikipedia. https://en.wikipedia.org/wiki/Lorentz transformation

Petrescu F.I., 2012, Cold nuclear fusion. Plasma Physics And Fusion Technology (S70), INIS 44(16).

\section{Nomenclature}

$\varepsilon_{0}=>$ the permissive constant (the permittivity)

$\varepsilon_{0}=8.85418 E-12\left[\frac{C^{2}}{N \cdot m^{2}}\right]$

$\mathrm{h}=>$ the Planck constant

$h=6.626 E-34[\mathrm{~J} \cdot \mathrm{s}]$

$\mathrm{q}=>$ electrical elementary load

for a deuteron $q_{1}=q_{2}=|e|=|-1.6021 E-19|[C]$

$\mathrm{c}=$ the light speed in vacuum

$c=2.997925 E 8\left[\frac{m}{s}\right]$

$m_{0}[\mathrm{~kg}]=>$ the rest mass of particle in movement

$m_{0}$ proton

$1.67262 \mathrm{E}-27[\mathrm{~kg}]$

$m_{0}$ electron

$9.11 \mathrm{E}-31[\mathrm{~kg}]$

$m_{0}$ deuteron

$3.34524 \mathrm{E}-27[\mathrm{~kg}]$ 